\title{
Making the Best of Mixed-Field Orientation of Polar Molecules: A Recipe for Achieving Adiabatic Dynamics in an Electrostatic Field Combined with Laser Pulses
}

\author{
Jens H. Nielsen, ${ }^{1}$ Henrik Stapelfeldt, ${ }^{2,3, *}$ Jochen Küpper, ${ }^{4,5,6, \dagger}$ Bretislav Friedrich, ${ }^{6}$ \\ Juan J. Omiste, ${ }^{7}$ and Rosario González-Férez ${ }^{7, \$}$ \\ ${ }^{1}$ Department of Physics and Astronomy, Aarhus University, 8000 Aarhus C, Denmark \\ ${ }^{2}$ Department of Chemistry, Aarhus University, 8000 Aarhus C, Denmark \\ ${ }^{3}$ Interdisciplinary Nanoscience Center (iNANO), Aarhus University, 8000 Aarhus C, Denmark \\ ${ }^{4}$ Center for Free-Electron Laser Science, DESY, 22607 Hamburg, Germany \\ ${ }^{5}$ Department of Physics, University of Hamburg, 22761 Hamburg, Germany \\ ${ }^{6}$ Fritz-Haber-Institut der Max-Planck-Gesellschaft, 14195 Berlin, Germany \\ ${ }^{7}$ Instituto Carlos I de Física Teórica y Computacional and Departamento de Física Atómica, Molecular y Nuclear, \\ Universidad de Granada, 18071 Granada, Spain
}

(Received 20 January 2012; published 7 May 2012)

\begin{abstract}
We have experimentally and theoretically investigated the mixed-field orientation of rotational-stateselected OCS molecules and achieved strong degrees of alignment and orientation. The applied moderately intense nanosecond laser pulses are long enough to adiabatically align molecules. However, in combination with a weak dc electric field, the same laser pulses result in nonadiabatic dynamics of the mixed-field orientation. These observations are fully explained by calculations employing both adiabatic and nonadiabatic (time-dependent) models.
\end{abstract}

DOI: 10.1103/PhysRevLett.108.193001

PACS numbers: 37.10.Vz, 33.15.-e, 33.80.-b, 42.50.Hz

Creating oriented samples of polar molecules, i.e., molecules with their dipole moment preferentially pointing towards one hemisphere rather than the opposite, has been a long-standing goal in molecular sciences. It was originally motivated by the crucial role played by orientation in chemical reaction dynamics [1]. More recently, its importance in novel applications such as (fs-time-resolved) photoelectron angular distributions [2-4], diffractionfrom-within [5], or high-order harmonic generation [6] has been recognized.

Early methods exploited purely electrostatic fields. Using an electric multipole focuser, molecules in a single low-field-seeking quantum state can be selected due to their first-order Stark effect [7-9]. The degree of orientation is determined, and also limited, by the selected state. Alternatively, a strong homogeneous electric field can create so-called brute-force orientation [10,11]. This method requires very high electric field strengths and works best for rotationally cold molecules with large permanent dipole moments.

In 1999 a method based on the combined action of a moderately intense, nonresonant laser field and an electrostatic field was proposed [12]. For the case that the laser field is turned on significantly more slowly than the rotational period(s) of the molecule adiabatic behavior was assumed. The time-independent calculations showed that the degree of orientation could be nearly perfect under conditions present in many experimental setups. Furthermore, the degree of alignment, i.e., the confinement of the molecular axes to space-fixed axes, could also be very high. In addition, the method should be generally applicable to a broad range of molecules and, therefore, promises the availability of strongly oriented and aligned molecules for various applications. Experiments performed in the first half of the 2000s showed the feasibility of the method but the degree of orientation observed was moderate $[13,14]$. A major reason for the weak orientation was that while the individual pendular states are strongly oriented, these states arise in pairs whose members are oriented oppositely with respect to one another. Consequently, the resulting overall degree of orientation, obtained as the weighted average over the populated quantum states, diminishes compared to what is expected for very cold or even single-state molecular ensembles. A significant improvement in the experimental capabilities was reported in 2009 when quantum-state selected molecules were employed as targets leading to strongly enhanced orientation [15-17]. However, it was already realized that an adiabatic description is not sufficient to reproduce the experimental observations [18].

In the present work, we seek the maximum of achievable orientation, as predicted by the original adiabatic description. Therefore, we prepare a nearly pure $\left(92_{-5}^{+3} \%\right)$ rotational-ground-state ensemble of OCS molecules [19] and use a laser pulse that is sufficiently strong to ensure sharp alignment and that is turned on a 100 times slower than the rotational period of the molecules. Our experimental observations are, however, at odds, both qualitatively and quantitatively, with the predictions of the original theory [12]. Instead, the experimental findings, exploring the dependence of the orientation on both the laser intensity and on the static field strength, can be 
rationalized by solving the time-dependent Schrödinger equation describing the mixed-field orientation. Our analysis directly shows the nonadiabatic coupling of the two sublevels of the near-degenerate doublets, created by the laser field, and allows us to predict the experimental conditions needed to ensure adiabatic dynamics.

The experimental setup has been described in detail before $[17,19,20]$ and only a few important details will be pointed out, see Fig. 1(a). A pulsed molecular beam is formed by expanding a mixture of 1 mbar of OCS and 10 bar of neon into vacuum through a pulsed valve. The molecular beam is skimmed twice before entering a 15 -cm-long electrostatic deflector. Here it is spatially dispersed in the vertical direction according to the effective dipole moments of the quantum states [17]. Hereafter, the molecules travel into a velocity map imaging (VMI) spectrometer where they are crossed by two pulsed laser beams. The first pulse $\left(\mathbf{E}_{\text {align }}, \lambda=1064 \mathrm{~nm}, \tau_{\mathrm{FWHM}}=8 \mathrm{~ns}\right.$, linearly polarized) provides the laser field for the mixed-field orientation whereas the weak static field, $\mathbf{E}_{\text {stat }}$, exploited for the orientation is (inherently) provided as part of the VMI spectrometer, which also defines its direction. The second pulse (probe, $\lambda=800 \mathrm{~nm}, \tau_{\text {FWHM }}=30 \mathrm{fs}$, linearly polarized) is used to characterize the orientation and alignment by multiply ionizing the molecules, this is followed by Coulomb explosion and imaging of the recoiling $\mathrm{S}^{+}$fragments on a two-dimensional detector.

The strongest orientation is expected when $\mathbf{E}_{\text {align }}$ is parallel to $\mathbf{E}_{\text {stat. }}$. This geometry is, however, not well suited for the ion imaging method to characterize the orientation because the experimental observable, the $\mathrm{S}^{+}$ions, will then be localized in the center of the detector. Consequently, all measurements are conducted with $\mathbf{E}_{\text {align }}$ rotated by an angle $\beta \neq 0$ with respect to $\mathbf{E}_{\text {stat }}$ [see Fig. 1(a)]. Figures 1(b) and 1(c) show examples of $\mathrm{S}^{+}$ion images for $\beta=45^{\circ}$ and $135^{\circ}$ (equal to $-45^{\circ}$ ). The $\mathrm{S}^{+}$ions from the Coulomb explosion channel $\mathrm{S}^{+}+\mathrm{CO}^{+}$, appearing in the outermost part of the images, are highly directional and provide direct information about the alignment and orientation of the OCS molecules at the time of ionization.

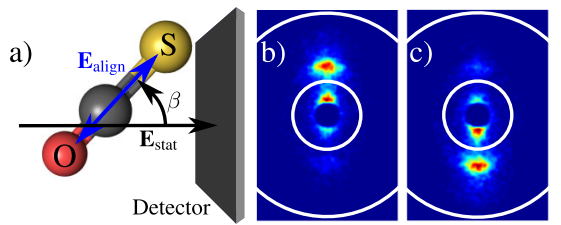

FIG. 1 (color online). (a) Schematic of the field configurations showing the polarization directions of the alignment and the probe pulses as well as the static field inside the VMI spectrometer and the definition of the angle $\beta$. (b), (c) $\mathrm{S}^{+}$ion images for $\beta=45^{\circ}$ and $135^{\circ}$, respectively, and $E_{\text {stat }}=571 \mathrm{~V} / \mathrm{cm}$. The rings in the image depict the limits for $\mathrm{S}^{+}$ions from the $\mathrm{S}^{+}+\mathrm{CO}^{+}$channel used in the calculation of the degree of orientation.
The strong angular confinement of the $\mathrm{S}^{+}$ions shows that the OCS molecules are sharply one-dimensionally aligned along $\mathbf{E}_{\text {align }}$. In addition, a pronounced asymmetry of the $\mathrm{S}^{+}$ions emitted either along or opposite to $\mathbf{E}_{\text {stat }}$, with an excess of $\mathrm{S}^{+}$in the upper (lower) region for $\beta=45^{\circ}\left(135^{\circ}\right)$ is observed. This shows that the molecules are oriented with the $\mathrm{S}$ end preferentially pointing toward the detector screen-as expected and in agreement with previous studies [21]. To quantify the degree of orientation only ions from the $\mathrm{S}^{+}+\mathrm{CO}^{+}$channel are considered. We then specify the orientation by the ratio $N_{\text {up }} / N_{\text {tot }}$ of the number of these ions in the upper half of the image $N_{\text {up }}$ compared to the total number of ions $N_{\text {tot }}$ from this channel.

In the first set of measurements the degree of orientation is recorded as a function of the alignment pulse intensity, $I_{\text {align }}$, for two values of $E_{\text {stat }}$. The results are shown in Fig. 2 For low values of $I_{\text {align }}$ the orientation ratio is almost the same for the two static fields but for $I_{\text {align }}>$ $2.5 \times 10^{11} \mathrm{~W} / \mathrm{cm}^{2}$ the results differ. For the strong static field the orientation reaches a maximum of approximately 0.8 at $I_{\text {align }}=5 \times 10^{11} \mathrm{~W} / \mathrm{cm}^{2}$ and remains essentially constant out to $1.4 \times 10^{12} \mathrm{~W} / \mathrm{cm}^{2}$. In contrast, for the smaller static field the maximum orientation occurs already at $I_{\text {align }}=3 \times 10^{11} \mathrm{~W} / \mathrm{cm}^{2}$ and the degree of orientation decreases as $I_{\text {align }}$ is further increased, dropping to 0.70 at $I_{\text {align }}=1.4 \times 10^{12} \mathrm{~W} / \mathrm{cm}^{2}$. The calculated degree of mixed-field orientation using the adiabatic model for $\beta=45^{\circ}$, as in the experiment, is shown in Fig. 2. Here, we have used rotational-state populations, in the coordinate system of the electric field in the deflector, with $92 \%$ in the $|\tilde{0}, \tilde{0}\rangle$ state, adiabatically corresponding to the field-free $J=M=0$ state, $4 \%$ in the $|\tilde{1}, \tilde{1}\rangle$ state and $4 \%$ in the $|\tilde{1}, \tilde{-1}\rangle$ state [19]. These states are projected onto a coordinate system for the mixed-field orientation that is defined by $\mathbf{E}_{\text {align }}$. The properly symmetrized states are then $|0,0, e\rangle$, $|1,1, e\rangle$, and $|1,1, o\rangle$, where $e$ and $o$ denote even and odd parity with respect to the plane defined by $\mathbf{E}_{\text {stat }}$ and $\mathbf{E}_{\text {align, }}$, respectively. The volume effect [18] is accounted for by using an experimentally determined cubic dependence on
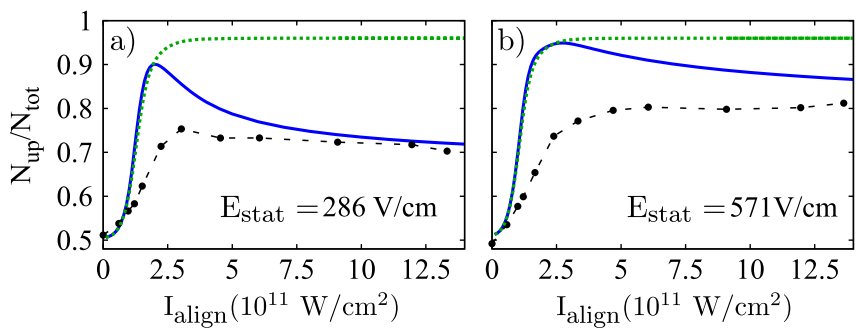

FIG. 2 (color online). Orientation ratio for $\beta=45^{\circ}$ as a function of $I_{\text {align }}$, for the weak (a) and the strong (b) static field, showing the experimental results (black solid circles), the adiabatic calculations (green dotted line) and the time-dependent calculations (blue solid line). 
the probe pulse intensity. These calculations predict that the orientation is independent of the applied static field. Following a rapid initial rise it reaches a value of 0.96 already at $4 \times 10^{11} \mathrm{~W} / \mathrm{cm}^{2}$ and remains constant. Clearly, these predictions are at odds with the experimental findings: The simulated degree of orientation is much too strong and it does not reproduce the decrease of the orientation at intensities above $3 \times 10^{11} \mathrm{~W} / \mathrm{cm}^{2}$, that is experimentally observed for the case of the smaller static field.

In the second set of measurements, shown in Fig. 3, the degree of orientation is recorded as a function of $\beta$ for the weak and the strong static fields and for a fixed value of $I_{\text {align }}$ of $9.1 \times 10^{11} \mathrm{~W} / \mathrm{cm}^{2}$. For both static field strengths $N_{\text {up }} / N_{\text {tot }}$ decreases monotonically as $\beta$ increases from $30^{\circ}$ to $150^{\circ}$. At all $\beta$ values the strong field leads to stronger orientation than the weak field. The $N_{\text {up }} / N_{\text {tot }}$ ratios calculated from the adiabatic model are essentially identical for the two static field strengths. The sharp rise (fall) of the curve to a value close to $0.96(0.04)$ as $\beta$ is increased (decreased) below (above) $90^{\circ}$ shows that very strong orientation is reached already for a very modest static electric field along $\mathbf{E}_{\text {align. }}$ This calculated behavior of the orientation differs qualitatively as well as quantitatively from the experimental results.

To obtain a better model of the mixed-field orientation process we solve the time-dependent Schrödinger equation using the experimental field configurations and rotationalstate populations [22]. The results for $N_{\text {up }} / N_{\text {tot }}$ as a function of $I_{\text {align }}$ are shown in Fig. 2. The predictions of stronger orientation for the strong static field and, in the weak static field case, the decreasing orientation at increasing intensity for $I_{\text {align }}>3 \times 10^{11} \mathrm{~W} / \mathrm{cm}^{2}$ are in line with the experimental findings. Moreover, the smooth $\beta$ dependence of the orientation, shown in Fig. 3, is fully captured by the time-dependent calculations. Quantitatively, the calculated values overestimate the degree of orientation. This could partly be due to temporal substructure in the experimentally applied laser pulses, which could induce more

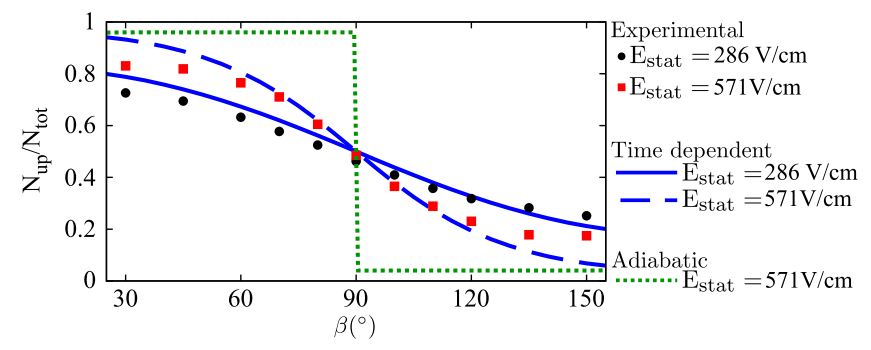

FIG. 3 (color online). The orientation ratio as a function of $\beta$ for $I_{\text {align }}=9.1 \times 10^{11} \mathrm{~W} / \mathrm{cm}^{2}$ for the experiment using $E_{\text {stat }}=286 \mathrm{~V} / \mathrm{cm}$ (black circles) and $\mathbf{E}_{\text {stat }}=571 \mathrm{~V} / \mathrm{cm}$ (red squares), for the adiabatic calculations, which are identical for the two static fields (green dotted line), and for the timedependent calculations using $E_{\text {stat }}=286 \mathrm{~V} / \mathrm{cm}$ (blue dashed line) and $E_{\text {stat }}=571 \mathrm{~V} / \mathrm{cm}$ (blue solid line). nonadiabatic population transfer. Overall, these non-adiabatic-model calculations are in much better agreement with the experimental results than the adiabatically calculated ones.

The underlying physical picture for understanding the failure of the adiabatic model is obtained by considering the evolution of the states during the turn-on of the alignment pulse. Before the pulse, the rotational states are essentially described by field-free rotor states. As the laser field strength increases, the states are hybridized by the combined action of the laser and static fields. In Fig. 4(a) the formation of doublets of nearly degenerate pendular states in the strong laser-field regime is shown. For the laser and static fields used in the experiment the absolute ground state $|0,0, e\rangle_{p}$ is right-way oriented; i.e., the permanent dipole moment is pointing along $\mathbf{E}_{\text {stat }}$. The upper level, $|1,1, e\rangle_{p}$, of the lowest doublet is wrong-way oriented-see Fig. 4(b).

As the alignment field is turned on the states that eventually form the near-degenerate doublet are coming closer together. This is illustrated in Fig. 4(a) for the $|0,0, e\rangle$, $|1,1, e\rangle$ pair and the $|1,0, e\rangle,|2,2, e\rangle$ pair and it results from the ac Stark interaction. When the energy splitting within a pair approaches the coupling strength due to the dc Stark interaction between the two sublevels, the two states in
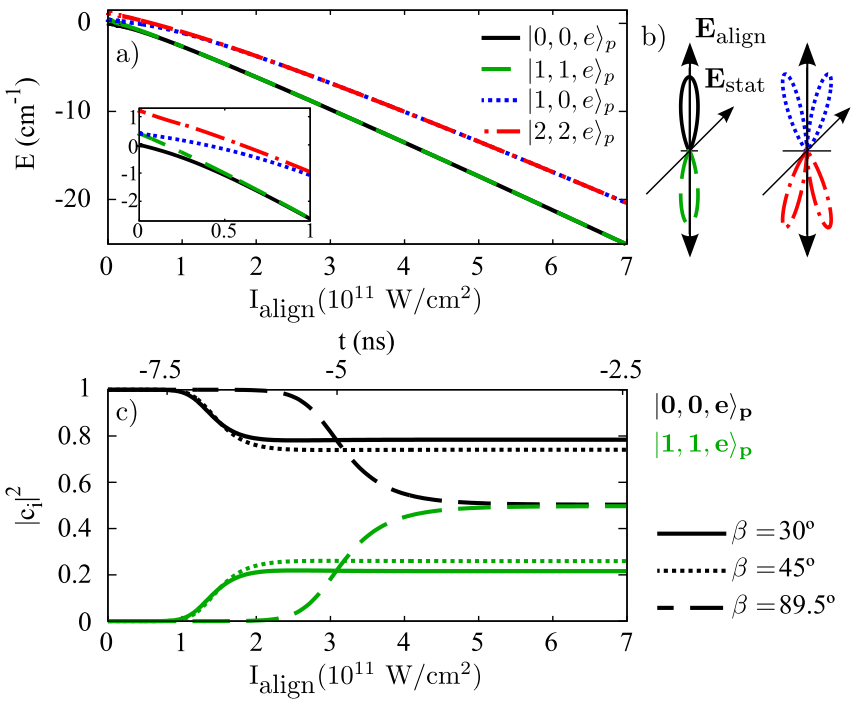

FIG. 4 (color online). (a) Energy of the four lowest lying rotational eigenstates as a function $I_{\text {align }}$ (time). The inset shows the relevant energy and intensity ranges where the formation of the near-degenerate doublets occurs. (b) Polar plot representation of the wave functions for the four states shown in (a) at $I_{\text {align }}=9 \times 10^{11} \mathrm{~W} / \mathrm{cm}^{2}$. The single-headed arrow shows the direction of the static field. (c) The squares of the coefficients for the projection of the time-dependent pendular wave function of the absolute ground state onto the adiabatic pendular state basis $\left[|0,0, e\rangle_{p}\right.$ (black) and $|1,1, e\rangle_{p}$ (green)] as a function of $I_{\text {align }}$ (time) for $\beta=30^{\circ}$ (solid line), $45^{\circ}$ (dotted line), and $89.5^{\circ}$ (dashed line). $E_{\text {stat }}=286 \mathrm{~V} / \mathrm{cm}$ for all data. 
each pair can mix because they have the same symmetry, provided the laser and static fields are nonperpendicular. This will result in population transfer between the oriented and antioriented states. The probability for mixing, corresponding to a crossing from one state of the doublet to the other, is determined by the rate of the turn-on and the energy separation between the $|0,0, e\rangle_{p}$ and $|1,1, e\rangle_{p}$ states. If the splitting is small, which is the case for a weak static electric field, the two states will be strongly coupled. To ensure fully adiabatic transfer it is necessary to turn on the laser field on a time scale slower than the inverse of the energy splitting of the near-degenerate doublet. This time can be much longer than the rotational period of the molecule. For the lowest doublet formed in OCS with $E_{\text {stat }}=286 \mathrm{~V} / \mathrm{cm}$ and $I_{\text {align }}=9.1 \times 10^{11} \mathrm{~W} / \mathrm{cm}^{2}$ our calculations show that the alignment pulse must be $50 \mathrm{~ns}$ long to ensure adiabatic transfer. The $8 \mathrm{~ns}$ pulses used in the current experiments do not fulfill this adiabaticity criterion although they are a hundred times longer than the rotational period of OCS - the condition previously considered sufficient for adiabatic behavior.

The population transfer is illustrated in Fig. 4(c), where the decomposition of the time-dependent state, which starts as the rotational ground state, in terms of the pendular states, is shown during the time interval representing the turn-on of the alignment pulse for three $\beta$ values. The fieldfree ground state is not transferred adiabatically to the pendular ground state: for $\beta=45^{\circ}$, the final state is decomposed into $74.06 \%|0,0, e\rangle_{p}$ and $25.94 \%|1,1, e\rangle_{p}$. Therefore, the resulting degree of orientation falls below that expected for a pure adiabatic transfer since the $|1,1, e\rangle_{p}$ state is wrong-way oriented. Similarly, other field-free rotational states are mixed with different pendular states during the turn-on, for instance the initial state $|\tilde{1}, \tilde{1}\rangle$ ends up in a superposition of $13.00 \%|0,0, e\rangle_{p}$, $37.10 \%|1,1, e\rangle_{p}, 35.64 \%|1,0, e\rangle_{p}$, and $14.26 \%|2,2, e\rangle_{p}$. For $\beta=89.5^{\circ}$, the electric field along the molecular axis is small, and the $|0,0, e\rangle_{p}$ and $|1,1, e\rangle_{p}$ states contribute with $50.32 \%$ and $49.68 \%$, respectively, to the time evolution of $|\tilde{0}, \tilde{0}\rangle$, resulting in a vanishing orientation. By contrast, alignment is expected to remain strong since both the $|0,0, e\rangle_{p}$ and $|1,1, e\rangle_{p}$ states imply tight confinement of the molecular axis along the laser-field polarization, see Fig. 4(b). The experimental observations for perpendicular fields do indeed show no orientation but strong alignment [23].

In summary, the combined action of a moderately strong laser field and a weak electrostatic field remains an attractive approach for creating tightly oriented molecules, but to fully exploit the potential of the method it is necessary to redefine the meaning of adiabatic conditions. Unlike alignment, where adiabaticity is ensured by turning on the laser field slower than the rotational period of the pertinent molecule, adiabatic transfer in orientation necessitates that the laser field be turned on slower than the inverse of the minimum spacing between the two pendular states in a doublet. This has repercussions for designing experimental parameters such that the degree of orientation be optimized. In the case of OCS, our calculations show that when $E_{\text {stat }}=286 \mathrm{~V} / \mathrm{cm}$ and $\beta=45^{\circ}$ adiabatic transfer of the $|\tilde{0}, \tilde{0}\rangle$ state to the $|0,0, e\rangle_{p}$ state is obtained if a transform-limited laser pulse with a Gaussian pulse duration (full width half maximum) of $50 \mathrm{~ns}$ is used. In practice, such pulses are not easily supplied by lasers typically present in laboratories. Considering instead the 10 ns output from the widespread Nd:YAG lasers adiabatic transfer will occur for a static electric field of $2 \mathrm{kV} / \mathrm{cm}$ : The increased static field leads to a larger minimum spacing of the doublet and, thus, relaxes the requirement for the slowness of the laser-field turn-on. Such static fields are compatible with, for instance, VMI spectrometers. For a pulse durations of $500 \mathrm{ps}$, which would be relevant for using the stretched output from amplified Ti-sapphire lasers, a static field of $10 \mathrm{kV} / \mathrm{cm}$ is needed to ensure adiabatic conditions. Again this is compatible with electron or ion spectrometers [16].

We note that the lack of adiabatic behavior will also be influenced by avoided crossings between rotationally excited states at low laser intensities. For larger molecules, where the rotational level structure is quite complex, this effect is expected to be particularly important $[18,24]$, but an increase of the static field strength should enhance the degree of orientation as already demonstrated experimentally for several asymmetric top rotors $[2,15,17,20]$.

Financial support by the Spanish Project No. FIS201124540 (MICINN) as well as Grant No. FQM-4643 (Junta de Andalucía), the Lundbeck Foundation, the Carlsberg Foundation, and the Danish Council for Independent Research is gratefully appreciated. J. J. O. acknowledges the support of ME under the program FPU. R.G.F. and J. J. O. acknowledge support from the Andalusian research group Grant No. FQM-207.

\footnotetext{
*Corresponding author. henriks@chem.au.dk

${ }^{\dagger}$ Corresponding author. jochen.kuepper@cfel.de Corresponding author. rogonzal@ugr.es
}

[1] P. Brooks, Science 193, 11 (1976).

[2] L. Holmegaard et al., Nature Phys. 6, 428 (2010).

[3] C.Z. Bisgaard, O. J. Clarkin, G. Wu, A. M. D. Lee, O. Geßner, C. C. Hayden, and A. Stolow, Science 323, 1464 (2009).

[4] J.L. Hansen, H. Stapelfeldt, D. Dimitrovski, M. Abusamha, C. P. J. Martiny, and L. B. Madsen, Phys. Rev. Lett. 106, 073001 (2011).

[5] A. Landers et al., Phys. Rev. Lett. 87, 013002 (2001).

[6] H.-J. Wörner (private communication). 
[7] J.P. Gordon, H. J. Zeiger, and C. H. Townes, Phys. Rev. 99, 1264 (1955).

[8] J. Reuss, in Atomic and Molecular Beam Methods, edited by G. Scoles (Oxford University Press, New York, 1988), Vol. 1, Chap. 11, pp. 276-292.

[9] T. P. Rakitzis, A. J. van den Brom, and M. H. M. Janssen, Science 303, 1852 (2004).

[10] H. J. Loesch and A. Remscheid, J. Chem. Phys. 93, 4779 (1990).

[11] B. Friedrich and D. R. Herschbach, Nature (London) 353, 412 (1991).

[12] B. Friedrich and D. Herschbach, J. Chem. Phys. 111, 6157 (1999).

[13] U. Buck and M. Fárník, Int. Rev. Phys. Chem. 25, 583 (2006).

[14] H. Sakai, S. Minemoto, H. Nanjo, H. Tanji, and T. Suzuki, Phys. Rev. Lett. 90, 083001 (2003).

[15] L. Holmegaard, J. H. Nielsen, I. Nevo, H. Stapelfeldt, F. Filsinger, J. Küpper, and G. Meijer, Phys. Rev. Lett. 102, 023001 (2009).

[16] O. Ghafur, A. Rouzée, A. Gijsbertsen, W. K. Siu, S. Stolte, and M. J. J. Vrakking, Nature Phys. 5, 289 (2009).
[17] F. Filsinger, J. Küpper, G. Meijer, L. Holmegaard, J. H. Nielsen, I. Nevo, J. L. Hansen, and H. Stapelfeldt, J. Chem. Phys. 131, 064309 (2009).

[18] J. J. Omiste, M. Gärttner, P. Schmelcher, R. GonzálezFérez, L. Holmegaard, J. H. Nielsen, H. Stapelfeldt, and J. Küpper, Phys. Chem. Chem. Phys. 13, 18815 (2011).

[19] J. H. Nielsen, P. Simesen, C. Z. Bisgaard, H. Stapelfeldt, F. Filsinger, B. Friedrich, G. Meijer, and J. Küpper, Phys. Chem. Chem. Phys. 13, 18971 (2011).

[20] J.L. Hansen et al., Phys. Rev. A 83, 023406 (2011).

[21] D. Dimitrovski, M. Abu-samha, L. B. Madsen, F. Filsinger, G. Meijer, J. Küpper, L. Holmegaard, L. Kalhøj, J.H. Nielsen, and H. Stapelfeldt, Phys. Rev. A 83, 023405 (2011).

[22] P. Sánchez-Moreno, R. González-Férez, and P. Schmelcher, Phys. Rev. A 76, 053413 (2007).

[23] L. Holmegaard, Ph.D. thesis, Aarhus University, 2010.

[24] J. Bulthuis, J. Möller, and H. J. Loesch, J. Phys. Chem. A 101, 7684 (1997). 